\title{
Barriers and Enablers of Weight Management After Breast Cancer: A Thematic Analysis of Free Text Survey Responses Using the COM-B Model
}

Carolyn Ee ( $\nabla$ c.ee@westernsydney.edu )

Western Sydney University

Freya MacMillan

Western Sydney University

John Boyages

ICON cancer centre

Kate McBride

Western Sydney University

\section{Research Article}

Keywords: breast cancer, weight, physical activity, qualitative, supportive care, COM-B

Posted Date: December 28th, 2021

DOI: https://doi.org/10.21203/rs.3.rs-1164503/v1

License: (a) (i) This work is licensed under a Creative Commons Attribution 4.0 International License. Read Full License 


\section{Abstract}

\section{Background}

Weight gain is common after breast cancer. The aim of this study was to identify and describe the barriers to and enablers of successful weight management for women with breast cancer.

\section{Methods}

This was a combined inductive and deductive framework analysis of free text responses to an anonymous cross-sectional survey on weight after breast cancer. Women were recruited mainly through the Breast Cancer Network Australia Review and Survey Group. We applied deductive thematic analysis to free text responses to questions on barriers, enablers, research priorities, and one open-ended question at the end of the survey using the Capability, Opportunity, Motivation and Behaviour (COM-B) model as a framework. Subthemes that arose from the inductive analysis were mapped onto the COM-B model framework. Findings were used to identify behaviour change intervention functions.

\section{Results}

133 women provided free text responses. Most women were of Caucasian origin and had been diagnosed with non-metastatic breast cancer, with a mean age of 59.1 years. Women's physical capability to adopt and sustain healthy lifestyle habits was significantly affected by treatment effects and physical illness, and some lacked psychological capability to self-regulate the face of stress and other triggers. Limited time and finances, and the social impact of undergoing cancer treatment affected the ability to control their diet. Frustration and futility around weight management were prominent. However, some women were confident in their abilities to self-regulate and self-monitor lifestyle behaviours, described support from friends and health professionals as enablers, and welcomed the physical and psychological benefits of being active in the context of embracing transformation and self-care after cancer.

\section{Conclusion}

Women need specific advice and support from peers, friends and families and health professionals. There is a gap in provision of supportive care to empower women to adopt and sustain healthy lifestyles. Environmental restructuring (including financial support), incentivization (creating an expectation of looking and feeling better), persuasion and coercion (aiming to prevent recurrence), and equipping women with specific knowledge and skills, would also facilitate optimal lifestyle behaviours and weight management.

\section{Background}

The most common cancer amongst women is breast cancer $(1,2)$ with the global incidence predicted to rise from 2 million new cases in 2018 to 3 million in 2040(1). Particularly for post-menopausal women, obesity or being overweight is a well-known risk factor(3). Obesity at diagnosis and weight gain after 
treatment has been linked to higher recurrence, breast cancer mortality and all-cause mortality rates $(2,4)$. Weight gain is a common occurrence after the diagnosis of breast cancer and has been linked to lower quality of life(2). Factors responsible for this weight gain include the use of chemotherapy, younger age at diagnosis, induced menopause, and reduction in physical activity $(2,5)$.

Given the growing population of breast cancer survivors and the link between weight gain and adverse health outcomes, research into weight management after breast cancer is of critical importance. An understanding of the barriers and enablers of successful weight management after breast cancer is needed in order to inform the development of appropriate interventions.

However, quantitative assessment lacks the richness and depth of qualitative evaluation and does not adequately capture the experience of weight management after breast cancer. Qualitative research seeks to understand the experiences and meaning in participants' lives, and can result in a deeper and more nuanced and comprehensive understanding of illness or behavior than quantitative research. The aim of this study was to identify and describe the barriers and enablers of successful weight management in women with breast cancer, using thematic analysis of 250 free text responses to our survey on weight management after breast cancer in women living in Australia (6). We used a theory-based approach to our analysis in order to fully understand the context in which weight loss behaviours (restricting diet and increasing physical activity) occur in our sample, by using the Capability Opportunity Motivation Behaviour (COM-B) theoretical model proposed by Michie et.al. (7). The COM-B model was developed after a comprehensive review of nineteen behaviour change frameworks and proposes that there are three essential components to any behaviour: capability (having the knowledge, skills and abilities to engage in a particular behaviour); opportunity (external factors that make a behaviour possible); and motivation (internal processes that influence decision making and behaviours). These form the hub of a "behaviour change wheel" around which are placed nine intervention functions that are aimed at addressing any gaps in capability, opportunity and motivation. Understanding behaviour within the framework of the COM-B therefore provides a foundation on which to select intervention strategies that can bring about behaviour change.

\section{Methods}

\section{Study design and inclusion criteria}

We conducted a cross-sectional, self-administered, anonymous survey using the online survey program Qualtrics (8) between November 2017 and March 2018. Ethics approval was granted by the Western Sydney University Human Research Ethics Committee (H12444, October 2017). Our methods have been previously described (6). Briefly, we recruited women mainly through the Breast Cancer Network Australia (BCNA) Review and Survey Group. BCNA is the largest breast cancer advocacy group in Australia. Limiting research at BCNA to the Review and Survey group allows researchers to access women who are engaged in the research process, while protecting the rest of BCNA from frequent research requests. Women were also recruited through online breast cancer support groups and women's health 
organisation social media pages in Australia. Any woman living in Australia who self-identified as having a breast cancer diagnosis was eligible to complete the survey. Participants were informed that the aim of the survey was to explore weight change after breast cancer. Participants were provided with an electronic copy of the Participant Information Sheet via a weblink on the survey website prior to commencing the survey, and were informed that consent was implied upon commencement.

\section{Data analysis}

Details of the survey instrument have been previously described (6). We conducted thematic analysis of free text responses to the questions outlined in Box 1. Three questions were multiple choice questions about barriers and enablers of successful weight loss and weight maintenance and research priorities for addressing weight concerns after breast cancer, and included a long free text option. The fourth question was a free text question that asked if survey participants had anything they would like to add. The thematic analysis approach was selected as it suits questions related to people's experiences, views or perceptions, and is a commonly used method for identifying, reporting and interpreting patterns within qualitative data (9).

\section{Box 1}

1. In your opinion, what are the barriers that you currently face or have faced in regards to successful weight loss: (please tick all that apply)

$\nabla$ Not enough time to exercise (please specify reason why e.g. family commitments, work commitments)

Not enough time to prepare healthier food options

$\nabla$ The financial cost (please specify)

$\nabla$ I have trouble keeping the weight off

\I don't get enough support from health professionals/family/friends (please specify)

$\otimes$ The information provided or programs available are not specific to breast cancer/cancer survivors

Q I feel that I don't have enough willpower/motivation

QI have or have had side effects from treatment

$\nabla$ Fatigue

QI have other health conditions/symptoms unrelated to cancer(please specify)

QI have other health conditions/symptoms related to cancer (please specify)

\Mental health reasons (stress, depression, anxiety, negative self talk, emotional eating, other) 
$\otimes I$ can't find reliable information

QI don't have someone to check in on how I am going with weight loss

\Other (long free text box)

2. What are some of things that have helped you lose weight and keep weight off? (please tick all that apply)

Q Being accountable to someone else

\Following a prescribed diet

\Social support - informal

\Social support - formal (e.g. a group)

\A Breast Cancer specific program (please specify)

$\nabla$ Support from a health professional (please specify)

\A structured exercise program

\Addressing my other symptoms e.g. lymphoedema hot flushes (please specify)

\Other (long text box)

3. In your opinion, what are the areas that researchers should focus on with regard to addressing weight concerns after breast cancer? (please tick all that apply)

Effectiveness of diet therapies

$\otimes$ Effectiveness of physical activity

$\nabla$ Medical treatments

$\nabla$ Surgical treatments

$\nabla$ Weight maintenance

Q Social support programs

\ Online programs

\Telephone-delivered programs

\ Other (please specify) 
4. Is there anything you would like to add? (Free text box)

Free text responses were retrieved from the online survey data and imported into Excel v16.55 software. Three researchers (KM, FM, CE) familiarised themselves with the open text responses. We used a framework analysis method (10), with participants as rows and themes and subthemes as columns. Two frameworks were developed, one for barriers and one for enablers of weight loss and weight loss behaviours. This method of analysis allowed us to identify consistent patterns and relationships within and across themes. The framework was developed both inductively (researcher-driven - KM and FM) (11) and deductively (using the COM-B model - CE and KM). The behaviours identified for the purposes of this study were controlling caloric intake and participating in physical activity. Where it was unclear which behaviour women were referring to in the free text responses, we coded the responses as "both [behaviours] or unclear". Each text response was first coded to whether it was a barrier or an enabler, then whether the participant was referring to restricting diet, increasing physical activity, or whether the text response referred to both or was unclear. The response was then coded using the COM-B model into the broad components of Capability, Opportunity or Motivation, and further coded into sub-components of Physical or Psychological Capability, Physical or Social Opportunity, and Reflective or Automatic Motivation. Last, the response was assigned a subtheme that arose from the inductive coding. Continued revision of the categories and emerging themes took place with the researchers searching for sub-topics and new insights into each category. The researcher used strategies such as independent coding, use of excerpts to support statements, and consensus meetings throughout to ensure that study inter-rater reliability and rigour were upheld by ensuring trustworthiness in coding.

Once this stage of coding was complete, we mapped the codes to intervention functions as described in the COM-B matrix by Michie et.al. Michie and colleagues identified nine intervention functions based on a comprehensive reivew of 19 behaviour change frameworks, each mapping on to one or more components of the COM-B model (7).

\section{Results}

A total of 309 women responded to the survey, of which 133 included 250 free text responses relevant to this study (Table 1). Most women were of Caucasian origin $(94.1 \%, n=144)$ with a mean age of 59.1 years ( $S D=9.1$, range 37-78, $n=128$ ). Most women who had provided a free text response had been diagnosed with non-metastatic breast cancer $(95.3 \%, 123 / 129)$ with an average of 7.9 years since diagnosis (SD 5.1, range 1-32 years, $n=130)$. The mean age at diagnosis was 51.3 years. Mean current BMI was $27.89 \mathrm{~kg} / \mathrm{m}^{2}$ (SD 6.1). Mean weight gain was $4.6 \mathrm{~kg}$ (SD 9.98, $n=127)$ and $55.1 \%(70 / 127)$ of this sample reported they had gained more than $5 \%$ of their body weight at diagnosis.

\section{Findings from the thematic analysis}

Themes and subthemes are described in Table 2 and additional quotes can be found in Additional Tables 1-6 [see Additional Tables]. Participants are identified by a unique ID number. 


\section{Table 2 Themes and subthemes}

\section{Capability - physical}

\section{Barriers}

By far the most prominent barrier faced by women in our study related to physical capability to exercise or control diet. These were both cancer-related (including treatment side effects) and caused by a wide range of non-cancer illnesses. For some, eating habits were affected by existing illness or symptoms from treatment. Many women attributed being menopausal and being on endocrine therapy as a cause for difficulty in maintaining a healthy weight. Women on endocrine therapy expressed frustration at not seeing results even with great effort, and at not being warned about the possibility of weight gain with endocrine therapy.

"Even though I no longer use it [tamoxifen], I still cannot lose weight unless I eat hardly anything" (ID 109)

Women reported often feeling fatigued, and experienced a wide range of other health problems like hypothyroidism and arthritis, which restricted their ability to maintain physical activity. Some women experienced aromatase-inhibitor induced arthralgia, which also impacted on their ability to exercise especially if it exacerbated existing arthritis or musculoskeletal problems.

Chemotherapy-induced peripheral neuropathy, as well as the interaction of chemotherapy with other medications already being taken for existing chronic conditions such as arthritis, also hindered being able to exercise. Ongoing effects from other treatment modalities were also reported to act as a barrier to exercise. For example, some participants described how their lung and cardiac function had been potentially affected by radiotherapy which in turn reduced their exercise capacity. Surgery also left its mark in other women with both lymphoedema and mastectomy surgical effects being reported as physical movement restrictions.

\section{Enablers}

Few women spoke of enablers for physical capability, except for one woman who described a benefit from being given specific exercises from an exercise physiologist.

\section{Capability - psychological}

\section{Barriers}

Some women described difficulty in self-regulating food or drink intake for reasons such as "stress, defiance but mostly enjoyment" (ID 11). They had difficulty regulating food intake in response to stress or physical illness, and this could be a result of a complex interplay of physical and mental processes, or due to a non-cancer reason such as work stress. One woman simply described one of her barriers as "Self-sabotage!!" (ID 74). 
"Coping with physical changes has been difficult, and coping with the after effects of cancer has left me troubled (eg. not being able to have children). Rightly or wrongly, I overindulge in food and alcohol, with no reason to stop" (ID 259).

Lack of support and specific information from healthcare professionals was another barrier to maintaining a healthy weight. Women described receiving conflicting advice from healthcare professionals, a failure to validate their concerns about weight gain, and vague, non-specific advice that was unhelpful.

"I just get a telling off whenever I go to my oncologist who simply suggests more exercise" (ID 8)

"My oncologist and breast care nurse never saw my weight gain as an issue (even when I raised my concerns). I felt discouraged by my treatment team, weight gain seemed a normal part of treatment and life after"( ID 62)

Enablers

Staying positive, creating good habits and being able to access specific programs or support from trained healthcare professionals (including exercise physiologists, general practitioners [GPs], nutritionists and psychologists) increased women's capability to manage their weight through diet and exercise. Women described using the skills of self-regulation and self-monitoring (often referred to as "willpower" and "accountability") and having a clear goal as enablers of weight loss and weight maintenance. As a result, this increased their confidence in being able to manage their weight. One woman described being able to move on from being a breast cancer survivor and being with "average people" as an enabler as it meant "I'm not treated as special. Just normal part of society!" and it had gotten her out of the "poor me, why me?" mindset (ID 219).

\section{Opportunity - physical}

\section{Barriers}

A range of environmental barriers were described which limited the time and energy available to maintain a healthy lifestyle. These included study, work and family commitments, or being in hot climates. Another barrier was not being able to afford services and programs that could help with weight maintenance, especially in the context of the financial burden imposed by having cancer.

"The cost of support programs adds additional financial strain; if there was a subsidised scheme for breast cancer patients it would be easier to tackle the issue and set realistic, achievable goals" (ID 19)

\section{Enablers}

Access to affordable programs, keeping high-calorie foods out of the house, and having a dog to walk to encourage physical activity were some of the enablers of diet and exercise. 


\section{Opportunity - social}

\section{Barriers}

Women described how the social impact of undergoing cancer treatment impacted on their ability to manage their diet. Friends and family "made meals for me that I would not normally eat" (ID 124) and there was an increase in social eating both during and after treatment. Some women also described their friends, family and healthcare professionals discouraging weight loss or not taking their weight gain seriously.

\section{Enablers}

Support from broad community networks appeared important, whether this involved providing and encouragement or motivating women to take part in group activities. Peer support, whether from people with cancer or people without, was a strong enabler of being able to maintain healthy lifestyle habits.

"There was also a Facebook private group where we could post our diets, what we were eating and get support with the other people taking part in the transformation. The support is wonderful" (ID 219)

\section{Motivation - automatic}

\section{Barriers}

Women described a "loss of willpower" and turning to comfort eating as a result of feeling depressed or weak following their treatment, while others described disliking exercise. Others identified fear of cancer recurrence as a barrier to weight maintenance.

"I think I have a sub conscience [sic] fear of weight loss as it has always meant the return of cancer or someone unwell, so as soon as I lose weight I put it back on by eating more! I realise this is irrational but it seems to happen every time!" (ID 100)

\section{Enablers}

Few women described enablers with regard to automatic motivation, with the exception of one woman who said she did not "like the feeling of my thighs rubbing so will lose weight until that happens" (ID 285).

\section{Motivation - reflective}

\section{Barriers}

Futility and frustration around weight management was a commonly reported barrier with several women believing there was little they could do about their weight loss with one woman stating that 'cancer patients get fat and stay fat'. Others described a love of food and cooking as a barrier to healthy eating, 
with one woman saying "life was too short" to be eating "hardly anything" to prevent weight gain (ID 109).

"I try programs but after a month of trying hard and not losing weight give up in frustration! Nothing has worked. Need to lose at least 5 kgs but won't budge. I get despondent" (ID 201)

\section{Enablers}

Some women had managed to embrace healthier lifestyles, motivated by the end of their treatment or even the experience of getting cancer itself. One woman described cancer as her "wake-up call" to focus on herself, and reported being the healthiest she had ever been (ID 158). Other women were clearly motivated by the physical and psychological benefits of being active believing these activities were integral to prevention of mental health and breast cancer treatment-related physical issues. Women were motivated by wanting to get fitter, look better and feel better, and avoiding cancer recurrence. A focus on healthy eating to prevent recurrence was identified as an enabler of motivation, as well as being told to lose weight by a trusted healthcare professional. Adopting healthier lifestyles was seen as a form of selfcare and transformation after cancer. Others wanted to know the cause of weight gain, and one woman noted that the fun and welcoming environment of her gym along with the financial and other incentives offered for achieving goals, was a strong motivator to maintain a healthy lifestyle.

"At the end of the 28 days there was an award for the person that conquered the most demons on their journey and 2 scholarship memberships on cheaper rate. And I won one!... There's a notice board with positive affirmations, there are little Buddhism sayings on the walls, relaxing pictures, the music is great, the instructors have positive and happy energy" (ID 219)

"I have started doing aqua Aerobics and Chiball so hopefully help my mind and of course my body, weight and aching bones!!.! have turned myself around" (ID 180)

\section{Intervention functions}

Figure 1 depicts the mapping of the intervention functions identified by Michie et. al. according to the COM-B components identified in our study. In order to maximise capability, women require training (attaining skills) how to self-regulate, set goals, and self-monitor their behaviour and education (provision of specific information on what exercises are beneficial and what foods they should eat). Maximising opportunity to perform weight loss behaviours requires environmental restructuring (including the provision of additional financial support, important in the context of the financial toxicity of cancer). Persuasion, coercion and incentivisation can be used to create a sense of reward from looking and feeling better, and motivate women to change behaviour with the aim of preventing recurrence of cancer. Last, enablement (beyond environmental restructuring, training and education) is required to optimise all components. This includes efforts to relieve physical symptoms, and provision of support from peers, social networks and healthcare professionals. 


\section{Discussion}

In this theory-based thematic analysis of free text responses to a national survey, we identified barriers and enablers of weight management after breast cancer and mapped these to intervention functions using a rigorous and comprehensive theoretical model as a framework. Ours is the first study to apply a COM-B based analysis of weight loss behavious in women with breast cancer. These findings provide an important foundation to underpin behaviour change interventions to assist women in preventing weight gain and/or achieving weight loss after breast cancer treatment.

In women with breast cancer, weight loss interventions combining diet and exercise interventions with behaviour modification have generally been shown to achieve modest weight losses $(12,13)$. A recent Cochrane review reported that ultimodal interventions appeared superior to interventions offering diet interventions alone (MD -2.88kg, 95\% $\mathrm{Cl}-3.98,1.77 \mathrm{~kg}$ ) for change in body weight and concluded that further research is required to determine optimal weight loss interventions for women with breast cancer (13). Given the importance of providing women with behavioural support to achieve sufficient weight loss together with lifestyle interventions, our findings make a significant contribution to informing the development of optimal behaviour change interventions.

Previous qualitative research has reported several barriers to weight loss after breast cancer, including physical (e.g. the ageing process), environmental/organisational (e.g. traditional female caregiver roles), and psychosocial barriers (e.g. dislike of the gym) $(14,15)$. Similarly, we have previously reported on barriers and facilitators of weight management from our national survey of women living with $B C$ in Australia (16). We identified the most common barriers to successful weight management as being lack of motivation, fatigue, and difficulty maintaining weight, consistent with findings from a recent scoping review (17). However, the current study is the first to systematically map these barriers and enablers using the COM-B theoretical model. This has practical significance as it facilitates structured mapping of these barriers and enablers to specific behaviour change strategies and techniques, based on the intervention functions identified, which we discuss below.

\section{Enablement}

A key intervention function identified through our analysis is enablement. Enablement of a behaviour refers to increasing the means to and/or reducing barriers to increasing capability (beyond education and training) or opportunity (beyond environmental restructuring). Enablement of weight loss behaviours in women with breast cancer requires a comprehensive approach incorporating supportive care, management of comorbidities, and support from peers, social networks, and healthcare professionals. We found the impact of physical illness, both cancer and non-cancer related, on women's ability to undertake weight loss behaviours was profound. These findings are consistent with a qualitative study of 17 women with breast cancer, the undesirable effects of cancer treatment on diet as well as physical activity were noted by participants (18), including fatigue, and dietary changes due to chemotherapy effects. 
Our findings highlight a gap in provision of adequate whole-person supportive care. Symptoms such as fatigue, pain, cognitive deficits and anxiety are common among cancer survivors and may present for years after treatment $(19,20)$. Cancer survivors report not being prepared of the health risks of the posttreatment phase (21). Oncology-led survivorship care is not sustainable due to increasing numbers of survivors (21). There is growing recognition that cancer survivorship must shift towards a chronic disease model, with primary care needing to play an increasingly larger role due to the significant burden on hospital-based care $(19,22)$. Randomised controlled trials have already demonstrated the safety and effectiveness of shared follow-up care (23). GPs are ideally placed to provide high-quality whole person care for survivors by providing lifestyle advice, assisting with symptoms management, comorbidities and psychosocial issues, as well as referral to multidisciplinary teams (eg dieticians, exercise physiologists, psychologists) and community-based programs (19).

Fatigue is common after cancer (24), with other studies reporting this as a common barrier to physical activity in BC survivors (25). We have also demonstrated lower physical activity levels in women who cited fatigue as a barrier to weight management (6), yet one of the most effective treatments for postcancer fatigue is exercise $(26,27)$. Prescribing exercise to people with cancer can improve cancer-related fatigue, quality of life and physical function (28). Exercise training was recommended by the 2018 American College of Sports Medicine Roundtable as a means to improve common cancer-related health outcomes including anxiety, depressive symptoms and fatigue (29). This underscores the need for enablement of these women to make sustainable changes to their physical activity, through alleviation of their physical symptoms, and also training required in altering physical activity according capability. Our findings provide further validation for the benefits of exercise prescription after cancer.

Further, healthcare professionals caring for women with cancer should acknowledge concerns about weight gain and be able to offer meaningful advice on how to approach weight management. We found the experience of weight gain in itself is a stressor, as have others. In one qualitative study, women with breast cancer expressed surprise and concern associated with changes to weight and diet (18). In another study of African-American women with breast cancer, participants reported any change in weight (gain or loss) as a stressor that caused psychological distress and health concerns, with frustration at lack of control (30). Support, acknowledgement and timely referral by healthcare professionals is key in enabling women to optimize their weight after breast cancer.

We have previously reported that informal social support was cited as the fourth most important enabler of successful weight management in women with breast cancer (6). Similarly, other studies describe support and positive family and social environments as a facilitator of weight loss in breast cancer survivors (31). A study of Turkish women with breast cancer reported that women identified a number of needs including that their spouse and family needed to also receive information on healthy living (32) and the importance of household members in dietary decision making was noted in another study (33). Efforts to raise awareness about the importance of healthy lifestyle habits after breast cancer should involve the woman's family and other support networks, to provide a supportive environment for the woman and increase chances of successful behaviour change. This is especially important in the context 
of the change in eating habits occurring with an increase in social encounters and provision of meals by others after a cancer diagnosis, described by some women in our study.

There is mounting evidence for peer support models in supporting healthy lifestyle behaviour change and maintenance for weight loss and disease management, particularly diabetes (34-37). Peer support is support received from someone with similar characteristics to a patient and experiential knowledge changing lifestyle behaviours. Although few peer support interventions specifically for women after breast cancer have been evaluated, evidence for this type of support for lifestyle behaviour change in general, suggest it warrants future investigation.

\section{Environmental restructuring}

In our study, women described a range of external competing priorities leading to a sense of overwhelment and inadequacy. Similarly, Befort et al. reported psychosocial factors associated with weight gain after breast cancer included relationship changes and financial stressors (38). A qualitative study of women with breast cancer who took part in a diet and exercise intervention found the logistics of fitting the lifestyle program into work and family was challengeing (39). Women in our study also described the financial burden of having to pay for additional programs and support, further exacerbated by the financial toxicity of cancer (40). This further underpins the need for both holistic physical and psychosocial care among womn with breast cancer with implemention of strategies such as financial support and advice as part of environmental restructuring to facilitate behaviour change.

\section{Education and training}

We also identified a unmet need for some women to gain skills in self-regulation. A number of women had already mastered skills such as goal-setting, self-regulation and monitoring, and creating good lifestyle habits. Others were unable to regulate their eating in response to stressors, and described a disinterest from healthcare professionals when they expressed concern about weight gain. This highlights the need for behavioural support to equip these women with the skills necessary to help them achieve their lifestyle and weight goals.

\section{Persuasion, coercion and incentivisation}

Many women in our study spoke about being motivated by looking and feeling better with physical activity, and by the desire to avoid recurrence of cancer. This is supported by evidence that physical activity alleviates depressive symptoms in women with breast cancer (41). Physical activity has also been found to be seen as a way to regain control and reduce distress after breast cancer (42). A review found the experience of physical activity was positive among women with breast cancer with multiple benefits, including empowerment and a sense of reclaiming health (43), consistent with findings from our study. To optimize this motivation, health professionals should encourage women with breast cancer to adopt healthy lifestyle habits both for weight management as well as additional benefits to mental health and prevention of recurrence.

\section{Strengths and limitations}


This paper has several strengths. The nature of the open ended questions facilitated a broad range of responses, with the large sample size (133 women and 250 responses) allowing us to be confident we had collected comprehensive information. We employed strategies to ensure trustworthiness to ensure coding of data was conducted with rigour. The use of a well-established and comprehensive theoretical model adds further rigour and impact to our analysis and findings. However, we acknowledge that indepth interviews are more likely to yield further depth in responses and that our findings should be triangulated with data from other sources. Although the sample was not particularly ethnically diverse, we are reasonably satisfied the responses reflect the views of a diverse population of women who have been diagnosed with breast cancer. Nonetheless, the survey was only offered in English so non-English speaking participants were excluded. Further, we did not specify weight loss behaviours (e.g. diet, exercise) when we asked women about barriers and enablers, and therefore it was challenging to interpret some of the free text responses.

\section{Conclusions}

Our theory-based analysis identified what needs to shift in order for women with breast cancer to perform weight loss behaviours of restricting caloric intake and increasing physical activity. Enablement via provision of support from peers, social networks and healthcare professionals, and comprehensive supportive care, impacts on all key components of behaviour change. When combined with environmental restructuring, incentivization (creating an expectation of looking and feeling better), persuasion and coercion (aiming to prevent recurrence), and equipping women with specific knowledge and skills, these intervention functions will expand women's capability, opportunity and motivation to undertake the lifestyle behaviours that can lead to optimal weight management after breast cancer.

\section{Declarations}

\section{Ethics approval and consent to participate}

A copy of the Participant Information Sheet was provided to all participants prior to commencing the survey, and informed consent was implied upon commencing the online anonymous survey. Ethics approval was provided by the Human Research Ethics Committee, Western Sydney University $(\mathrm{H} 12444$, Oct 2017) and the study was performed in accordance with the Declaration of Helsinki.

\section{Consent for publication}

Not applicable

\section{Availability of data and materials}

The datasets used and/or analysed during the current study are available from the corresponding author on reasonable request. 


\section{Competing interests}

The authors declare that they have no competing interests.

\section{Funding}

This study did not receive any funding. CE's academic role is supported by an endowment from the Jacka Foundation of Natural Therapies.

\section{Authors' contributions}

CE conceived of the study, designed the survey instrument, and collected the data. JB contributed to design of the survey instrument and study. CE, FM and KM conducted the data analysis. CE, FM, JB and $\mathrm{KM}$ contributed significantly to interpretation of the data, drafting of the manuscript, and critical revision for important intellectual content and provided final approval for submission.

\section{Acknowledgements}

We thank the consumer representatives from Breast Cancer Network Australia who provided feedback on the survey instrument used in this study. Participants in this research were recruited from Breast Cancer Network Australia's (BCNA) Review and Survey Group, a national, online group of Australian women living with breast cancer who are interested in receiving invitations to participate in research. We acknowledge the contribution of the women involved in the Review and Survey Group who participated in this project. We also thank Karen Monaghan who assisted with some of the initial coding.

\section{References}

1. Bray F FJ, Soerjomataram I, Siegel RL, Torre LA, Jemal A. Global Cancer Statistics 2018: GLOBOCAN estimates of incidence ad mortality worldwide for 36 cancers in 185 countries. . CA Cancer J Clin, in press.

2. Vance V, Mourtzakis M, McCargar L, Hanning R. Weight gain in breast cancer survivors: prevalence, pattern and health consequences. Obesity reviews : an official journal of the International Association for the Study of Obesity. 2011;12(4):282-94.

3. Moley KH, Colditz GA. Effects of obesity on hormonally driven cancer in women. Science Translational Medicine. 2016;8(323).

4. Ewertz $M$, Jensen $M-B$, Gunnarsd $\sqrt{ } \geq t$ tir $K n \AA ⿻, 1 \sqrt{ } \prod$ jjis I, Jakobsen $E H$, Nielsen $D$, et al. Effect of Obesity on Prognosis After Early-Stage Breast Cancer. Journal of Clinical Oncology. 2011;29(1):2531.

5. Saquib N, Flatt SW, Natarajan L, Thomson CA, Bardwell WA, Caan B, et al. Weight gain and recovery of pre-cancer weight after breast cancer treatments: evidence from the women's healthy eating and living (WHEL) study. Breast cancer research and treatment. 2007;105(2):177-86. 
6. Ee C, Cave AE, Naidoo D, Bilinski K, Boyages J. Weight before and after a diagnosis of breast cancer or ductal carcinoma in situ: a national Australian survey. BMC Cancer. 2020;20(1):113.

7. Michie S, van Stralen MM, West R. The behaviour change wheel: a new method for characterising and designing behaviour change interventions. Implementation science : IS. 2011;6:42-.

8. Qualtrics. Qualtrics. Provo, Utah, USA2018.

9. Braun V, Clarke V. Successful Qualitative Research. Thousand Oaks: Sage; 2013.

10. Ritchie J. Lewis J (eds.): Qualitative Research Practice: A guide for Social Science Students and Researchers. London: Sage Publications; 2003.

11. Braun V, Clarke V. Using thematic analysis in psychology. Qualitative Research in Psychology. 2006;3:77-101.

12. Reeves MM, Terranova CO, Eakin EG, Demark-Wahnefried W. Weight loss intervention trials in women with breast cancer: a systematic review. Obesity reviews : an official journal of the International Association for the Study of Obesity. 2014;15(9):749-68.

13. Shaikh H, Bradhurst P, Ma LX, Tan SY, Egger SJ, Vardy JL. Body weight management in overweight and obese breast cancer survivors. Cochrane Database Syst Rev. 2020(12).

14. Brunet J, Taran S, Burke S, Sabiston CM. A qualitative exploration of barriers and motivators to physical activity participation in women treated for breast cancer. Disability and rehabilitation. 2013;35(24):2038-45.

15. Hefferon K, Murphy H, McLeod J, Mutrie N, Campbell A. Understanding barriers to exercise implementation 5-year post-breast cancer diagnosis: a large-scale qualitative study. Health education research. 2013;28(5):843-56.

16. Ee C, Cave AE, Naidoo D, Bilinski K, Boyages J. Weight management barriers and facilitators after breast cancer in Australian women: a national survey. BMC women's health. 2020;20(1):140.

17. Elshahat S, Treanor C, Donnelly M. Factors influencing physical activity participation among people living with or beyond cancer: a systematic scoping review. International Journal of Behavioral Nutrition and Physical Activity. 2021;18(1):50.

18. Kwok A, Palermo C, Boltong A. Dietary experiences and support needs of women who gain weight following chemotherapy for breast cancer. Support Care Cancer. 2015;23(6):1561-8.

19. Jefford M, Koczwara B, Emery J, Thornton-Benko E, Vardy J. General practice care of cancer survivors. Australian Journal for General Practitioners. 2020;49:288-92.

20. Tan SY, Turner J, Kerin-Ayres K, Butler S, Deguchi C, Khatri S, et al. Health concerns of cancer survivors after primary anti-cancer treatment. Support Care Cancer. 2019;27(10):3739-47.

21. Alfano CM, Jefford M, Maher J, Birken SA, Mayer DK. Building Personalized Cancer Follow-up Care Pathways in the United States: Lessons Learned From Implementation in England, Northern Ireland, and Australia. Am Soc Clin Oncol Educ Book. 2019;39:625-39.

22. Emery JD, Shaw K, Williams B, Mazza D, Fallon-Ferguson J, Varlow M, et al. The role of primary care in early detection and follow-up of cancer. Nature Reviews Clinical Oncology. 2014;11(1):38-48. 
23. Zhao Y, Brettle A, Qiu L. The Effectiveness of Shared Care in Cancer Survivors-A Systematic Review. Int J Integr Care. 2018;18(4):2.

24. Servaes $P$, Verhagen $C$, Bleijenberg $G$. Fatigue in cancer patients during and after treatment: prevalence, correlates and interventions. European journal of cancer (Oxford, England : 1990). 2002;38(1):27-43.

25. Ventura EE, Ganz PA, Bower JE, Abascal L, Petersen L, Stanton AL, et al. Barriers to physical activity and healthy eating in young breast cancer survivors: modifiable risk factors and associations with body mass index. Breast cancer research and treatment. 2013;142(2):423-33.

26. Juvet LK, Thune I, Elvsaas IKO, Fors EA, Lundgren S, Bertheussen G, et al. The effect of exercise on fatigue and physical functioning in breast cancer patients during and after treatment and at 6 months follow-up: A meta-analysis. Breast (Edinburgh, Scotland). 2017;33:166-77.

27. Meneses-Echavez JF, Gonzalez-Jimenez E, Ramirez-Velez R. Effects of supervised exercise on cancer-related fatigue in breast cancer survivors: a systematic review and meta-analysis. BMC Cancer. 2015;15:77.

28. Clinical Oncology Society of Australia. COSA Position Statement on Exercise in Cancer Care. Sydney, Australia; 2018 April 2018.

29. Campbell KL, Winters-Stone KM, Wiskemann J, May AM, Schwartz AL, Courneya KS, et al. Exercise Guidelines for Cancer Survivors: Consensus Statement from International Multidisciplinary Roundtable. Med Sci Sports Exerc. 2019;51(11):2375-90.

30. Halbert CH, Weathers B, Esteve R, Audrain-McGovern J, Kumanyika S, DeMichele A, et al. Experiences with weight change in African-American breast cancer survivors. The breast journal. 2008;14(2):1827.

31. Terranova CO, Lawler SP, Spathonis K, Eakin EG, Reeves MM. Breast cancer survivors' experience of making weight, dietary and physical activity changes during participation in a weight loss intervention. Support Care Cancer. 2017;25(5):1455-63.

32. Cebeci F, Yangin HB, Tekeli A. Life experiences of women with breast cancer in south western Turkey: a qualitative study. European journal of oncology nursing : the official journal of European Oncology Nursing Society. 2012;16(4):406-12.

33. Klassen AC, Smith KC, Shuster M, Coa KI, Caulfield LE, Helzlsouer KJ, et al. "We're Just Not Prepared for Eating Over Our Whole Life": A Mixed Methods Approach to Understanding Dietary Behaviors Among Longer Term Cancer Survivors. Integrative cancer therapies. 2018;17(2):350-62.

34. Fisher EB, Boothroyd RI, Elstad EA, Hays L, Henes A, Maslow GR, et al. Peer support of complex health behaviors in prevention and disease management with special reference to diabetes: systematic reviews. Clin Diabetes Endocrinol. 2017;3:4.

35. Ginis KA, Nigg CR, Smith AL. Peer-delivered physical activity interventions: an overlooked opportunity for physical activity promotion. Transl Behav Med. 2013;3(4):434-43.

36. Leahey TM, Wing RR. A randomized controlled pilot study testing three types of health coaches for obesity treatment: Professional, peer, and mentor. Obesity (Silver Spring, Md). 2013;21(5):928-34. 
37. Patil SJ, Ruppar T, Koopman RJ, Lindbloom EJ, Elliott SG, Mehr DR, et al. Effect of peer support interventions on cardiovascular disease risk factors in adults with diabetes: a systematic review and meta-analysis. BMC public health. 2018;18(1):398.

38. Befort CA, Klemp JR, Austin HL, Perri MG, Schmitz KH, Sullivan DK. Outcomes of a weight loss intervention among rural breast cancer survivors. Breast cancer research and treatment. 2012;132.

39. Balneaves LG, Van Patten C, Truant TL, Kelly MT, Neil SE, Campbell KL. Breast cancer survivors' perspectives on a weight loss and physical activity lifestyle intervention. Support Care Cancer. 2014;22(8):2057-65.

40. Yousuf Zafar S. Financial Toxicity of Cancer Care: It's Time to Intervene. JNCl: Journal of the National Cancer Institute. 2015;108(5).

41. Carayol M, Bernard P, Boiché J, Riou F, Mercier B, Cousson-Gélie F, et al. Psychological effect of exercise in women with breast cancer receiving adjuvant therapy: what is the optimal dose needed? Annals of Oncology. 2013;24(2):291-300.

42. Maley M, Warren BS, Devine CM. A second chance: meanings of body weight, diet, and physical activity to women who have experienced cancer. Journal of nutrition education and behavior. 2013;45(3):232-9.

43. Browall M, Mijwel S, Rundqvist $\mathrm{H}$, Wengstrom Y. Physical Activity During and After Adjuvant Treatment for Breast Cancer: An Integrative Review of Women's Experiences. Integrative cancer therapies. 2018;17(1):16-30.

\section{Tables}

Table 1 Demographic characteristics of survey respondents who provided a free text response 


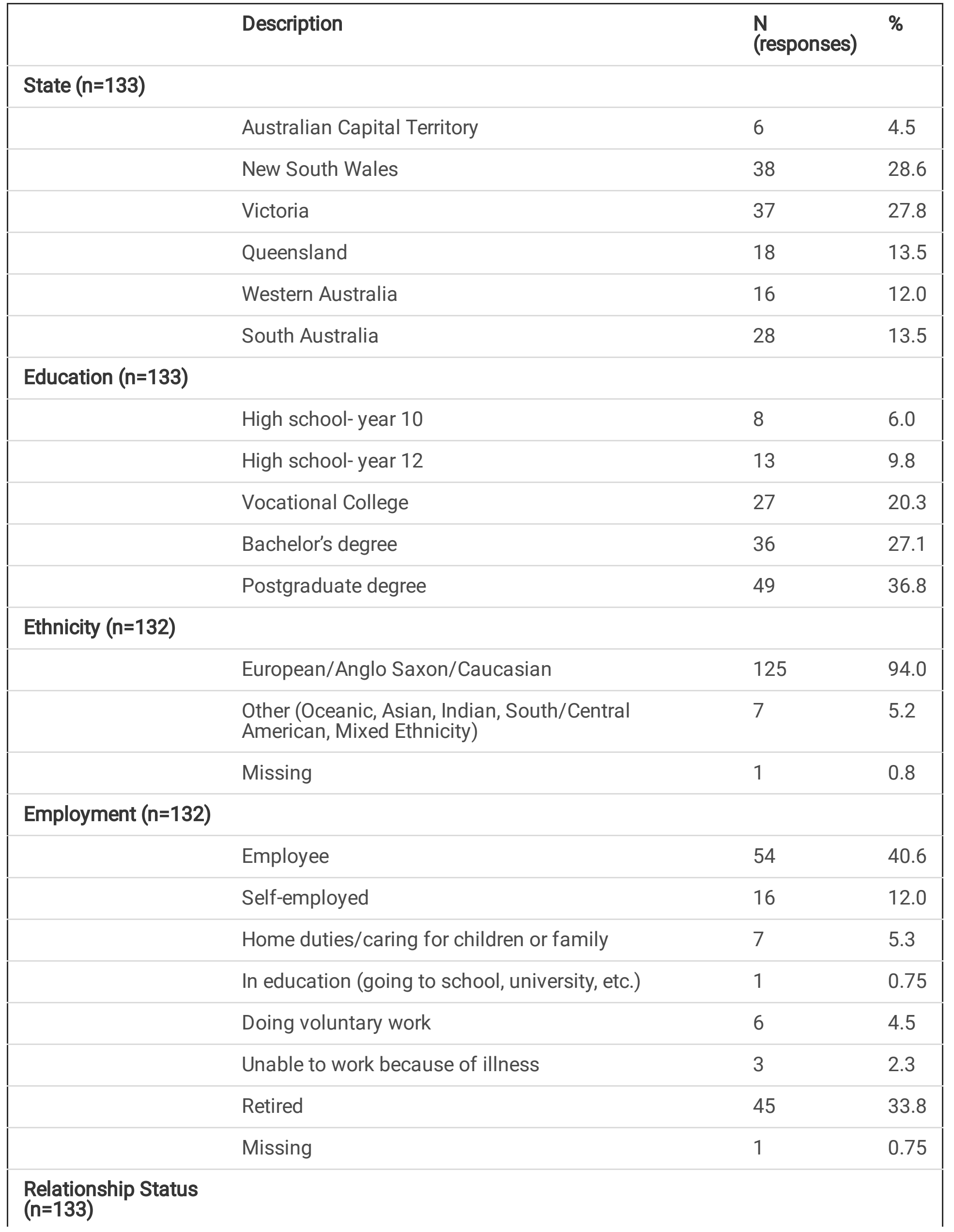




\begin{tabular}{|llll|}
\hline Single & 17 & 12.8 \\
\hline Married/de facto (living with partner) & 100 & 75.2 \\
\hline In a relationship (not living with partner) & 4 & 3.0 \\
\hline & Divorced/separated & 8 & 6.0 \\
\hline & Widowed & 4 & 3.0 \\
\hline $\begin{array}{l}\text { Weight gain pattern } \\
(\mathbf{n = 1 3 2 )}\end{array}$ & & & \\
\hline & Gained weight overall & 80 & 60.2 \\
\hline & Lost weight overall & 18 & 13.5 \\
\hline Weight remained stable & 20 & 15.0 \\
\hline Weight fluctuated a great deal & 14 & 10.5 \\
\hline Missing & 1 & 0.8 \\
\hline
\end{tabular}

Table 2 Themes and subthemes 


\section{Capability - attribute of a person makes a behaviour possible or facilitates it (together with} opportunity); capacity to carry out a behaviour.

Physical - capability that involves a person's physical function, skill, strength or stamina
Diet

Limited food options due to other health conditions

Symptoms from

treatment affects eating habits

\section{Exercise}

Physical illness makes exercise difficult (both cancer and non-cancer related)

\section{Both/unclear}

Menopause, physical illness and endocrine therapy makes weight loss difficult

Psychological - Knowledge or psychological skills, strength or stamina to engage in the necessary mental processes
Diet

Lack of interest/vague advice from health professionals

Unable to regulate eating in response to reasons apart from hunger

"Self sabotage"

Both/unclear

Distress

Lack of information

\section{Exercise}

Given specific exercises to use by a trained professional
Diet

Self-regulation

Specific information about diet (including doing own research)

Exercise

Creating good habits

Both/unclear

Specific program and support

Having a clear goal

Psychological support, positive mindset

Self-regulation and monitoring

Self-efficacy

Opportunity - attribute of an environmental system that makes a behaviour possible or facilitates it, together with capability 
Physical - Opportunity afforded by the environment, including time, resources, locations, cues, physical "affordance"
Diet

Availability of high calorie foods

Exercise

Environment (heat)

Exercise and both/unclear

Lack of time due to study/work/family commitments, general overwhelm

Financial cost

Social - Opportunity afforded by interpersonal influences, social cues and cultural norms that influence the way that we think about things

\section{Diet}

Other people

cooking/social eating

Unclear

Lack of support from

friends/health

professionals

Medical advice/social pressure to not lose too much weight
Diet

Limiting access to high calorie foods

Exercise

Having a dog to walk (ID 283)

Both

Affordable programs

\section{Exercise}

Peer support or support from family/friends

Both/unclear

Feeling normal again

Individualised approach

\section{Motivation - a mental process that energises and directs behaviour}

Reflective - involves plans (self-conscious intentions) and evaluations (beliefs about what is good or bad)
Diet

Enjoyment (or dislike) of food and cooking

Both/unclear

Beliefs that she cannot lose the weight

Frustration at not being able to lose weight

\section{Exercise}

Financial and other incentives, fun and welcoming environment

Helps mind and body

Look better, feel better

Both/unclear

Knowing the cause of weight gain

Wanting to avoid recurrence

Wanting to get fitter

Told to lose weight by someone she 
trusts

Cancer is a wake-up call

Automatic - involves emotional reactions, desires (wants and needs), impulses,

inhibitions, drive states and reflex responses

Diet:

Eating/drinking for reasons apart from hunger

Exercise: Dislikes

exercise

Both/unclear: Fear of recurrence

\section{Figures}

\section{Figure 1}

\section{Mapping of intervention functions to COM-B components}

\section{Supplementary Files}

This is a list of supplementary files associated with this preprint. Click to download.

- AdditionalTables.docx 